\title{
Chronic obstructive pulmonary disease: management of chronic disease
}

*Kirsty Hambleton, Clinical Fellow and Specialist Respiratory Trainee, Respiratory Medicine Unit, University of Oxford.

*Mona Bafadhel, Senior Clinical Researcher and Consultant Respiratory Physician, Respiratory Medicine Unit, University of Oxford.

Richard Russell

*No conflicts of interests related to this work.

\begin{abstract}
Chronic obstructive pulmonary disease (COPD) is the third leading cause of death worldwide, affecting an estimated 3 million people in the UK. The most common cause is tobacco smoke. Patients with COPD experience a high symptom burden, worsened during disease instability (termed exacerbations or 'lung attacks'), and a multidisciplinary approach should be adopted to manage this chronic lung disease. Diagnosis requires clinical and functional assessment to tailor treatments towards symptoms; the most common and debilitating of these are breathlessness, cough and sputum production. Breathlessness develops as a result of irreversible airway narrowing (obstruction), and spirometry is used alongside imaging to guide both diagnosis and treatment. To date, smoking cessation is the single most important intervention in delaying disease progression and should be a focus at every patient interaction. COPD is treated by a combination of pharmacological and non-pharmacological treatments, including pulmonary rehabilitation and self-management plans, allowing control over some of the symptom burden. Holistic management in COPD requires effective communication between all those involved in patient care, crossing secondary and primary care boundaries.
\end{abstract}

Keywords

Chronic obstructive pulmonary disease; multidisciplinary team; pulmonary rehabilitation; smoking cessation 


\section{Introduction}

Chronic obstructive pulmonary disease (COPD) is the third leading cause of death worldwide (World Health Organization, 2014) and the fifth highest in the UK. Approximately 1 million people in the UK have a diagnosis of COPD, with an estimated further 3 million of the population undiagnosed (British Lung Foundation, 2007)

The disease occurs most commonly as a result of cigarette smoking, including second-hand smoke, but specific occupational exposure including biofuels can be a contributory cause. COPD is progressive, with irreversible lung damage leading to loss of lung function, physical decline and dependence on health and social care services. COPD is often punctuated by periods of worsening symptoms, termed exacerbations or 'lung attacks'. Exacerbations account for 1 in 8 of all emergency hospital admissions and 1 million 'bed-days' each year, which costs the British National Health Service $\sim f 500$ million per year (National Institute for Clinical Excellence 2010, guidance cg101). The main aim of both secondary and primary care, through both pharmacological and non-pharmacological interventions, is to reduce and control exacerbations, and thus improve symptom burden and quality of life for patients with COPD. This heterogeneous disease requires individualized treatment incorporating a wide multidisciplinary team of clinicians, specialist nurses, physiotherapists, pharmacists, nutritionists, psychologists and palliative care.

\section{Diagnosis}

COPD is a broad disease label that encompasses several symptoms, patients often presenting with a combination of dyspnoea, predominately on exertion, cough and sputum production. Tobacco smoke is the most common cause, but patients should be asked about exposure to other inhaled substances, such as cannabis and biomass fumes. Other symptoms, such as weight loss, waking at night, ankle swelling and fatigue, should also be considered. Classification of symptoms using the Medical Research Council Dyspnoea Scale (Table 1) or COPD Assessment Test ${ }^{\mathrm{TM}}$ can be helpful in monitoring symptom progression (Figure 1).

Examination of patients with COPD may reveal few clinical signs, but the following should be recorded: presence or absence of cachexia, cyanosis, chest hyperinflation (Figure 2), pursed lip breathing, use of accessory muscles with respiration, and signs consistent with right heart failure and cor pulmonale, such as peripheral oedema and an elevated jugular venous pressure.

\section{Investigations}

COPD is characterized by irreversible airflow obstruction and classified using spirometry, measuring the post-bronchodilator forced expiratory volume in 1 second (FEV 1 ) and forced vital capacity (FVC). E a post-bronchodilator $\mathrm{FEV}_{1} / \mathrm{FVC}$ ratio $<0.7$ is consistent with a diagnosis of airflow obstruction. Disease severity is then classified according the $\mathrm{FEV}_{1}$ deficit (Table 2).

To further manage and investigate patients with stable COPD, other routinely available investigations are necessary. These include: pulse oximetry, an oxygen saturation $<92 \%$ saturation warranting referral for arterial blood sampling and a specialist oxygen assessment; a chest radiograph, to investigate the presence of bullae; and a full blood count to determine the presence of polycythaemia, which may merit referral on to secondary care for more detailed assessment. An $\alpha_{1}$ antitrypsin concentration is warranted in all patients who present at a young age, have a positive family for this history or have a minimal smoking history (<20 pack years). In difficult-to-manage patients, where the diagnosis is uncertain or where there are significant exacerbations and symptoms, further specialist tests include: imaging (computed tomography; Figure 3); sputum culture for detailed microbiological sampling; full pulmonary function tests, which are typically associated with an 
elevated residual volume and reduced carbon monoxide diffusion capacity; and an echocardiogram to specifically assess elevated pulmonary artery pressure and right ventricular dysfunction.

\section{Management of COPD}

In stable disease, treatment focuses on reducing frequency of exacerbations and slowing disease progression. This is most effectively achieved through a combination of pharmacological and nonpharmacological therapies. These goals have been prioritized by both the National Institute of Health and Care Excellence COPD guidelines as well as the international Global Initiative for Chronic Obstructive Lung Disease 2013 recommendations. The latest revision involves classification of patients according to a multidimensional assessment, taking into consideration lung function, risk of exacerbation and symptom burden (Figure 4). This approach is intuitive for physicians and patients alike, but is yet to be underpinned by a robust evidence base demonstrating efficacy and further work is required to evaluate this.

\section{Pharmacological therapies}

Inhaled therapy: the mainstay of inhaled treatment for COPD is maximal bronchodilation using both short- and long-acting $\beta_{2}$-adrenoreceptor agonists and anti-muscarinic agents, in addition to antiinflammatories in the form of inhaled corticosteroids. Decisions on treatment relate to symptom burden, exacerbations and lung function (Figure 5). Treatment is often given in a stepwise approach, with most patients ultimately requiring triple inhaled therapy.

Emerging evidence has demonstrated distinct inflammatory phenotypes of COPD [1] that could ultimately define tailored treatment for COPD; however, large clinical trials demonstrating this are still required.

Additional therapy: oral theophylline can be used in its slow-release form as add-on therapy in patients with COPD who continue to have symptoms despite inhaled therapy. Serum theophylline concentrations should be checked 3-5 days after starting treatment and then one week later. If stable they should be checked six-monthly. These medications are affected by cytochrome P450 enzyme inducers.

Oral mucolytic therapy can be trialed in patients with chronic productive cough and a high sputum load. They can help sputum expectoration and are often well tolerated.

There is evidence to show that in patients with severe COPD and frequent exacerbations lowdose macrolide therapy is effective in reducing exacerbation frequency[2]; but the mechanisms behind this oral agent are still not understood. At this stage, low dose macrolide therapy cannot be recommended as add-on therapy outside secondary care.

Oxygen therapy: long-term oxygen therapy should be considered for patients with COPD who have a resting arterial partial pressure of Oxygen $\left(\mathrm{PaO}_{2} \leq 7.3 \mathrm{kPa}\right.$ or $\mathrm{PaO}_{2} \leq 8 \mathrm{kPa}$ with evidence of peripheral oedema, polycythaemia or pulmonary hypertension with a recommended minimum of 15 hours per day (British Thoracic Society Home Oxygen Guidelines, 2015).

Non-invasive ventilation: in stable disease (i.e. not during an exacerbation), nocturnal non-invasive ventilation may be necessary in patients with COPD who develop respiratory acidosis and/or have a rising $\mathrm{PaCO}_{2}$ while on long-term oxygen therapy. NIV may confer a survival benefit in patients with stable hypercapnic disease; but any form of NIV should be managed by secondary care teams. 
Thoracic surgery/bronchoscopic implant techniques: in selected patients with severe emphysema, lung volume reduction surgery and/or bullectomy may be appropriate [3] . Endobronchial valves and coils (bronchoscopic lung volume reduction) may have short-term benefits of lung function and exercise capacity in carefully selected patients [4].

\section{Non-pharmacological therapies}

The impact of these therapies should not be underestimated, as a breadth of evidence shows that such interventions have the greatest impact on impacting on disease progression and maintaining physical function.

Smoking cessation: almost $20 \%$ of the UK population smoke, which remains a concerning statistic. Smoking cessation is the single most important factor in slowing disease progression, in addition to reducing symptom [5] in patients with COPD. Smoking cessation support should be offered to all continuing smokers through referral to smoking cessation services, where behavioral support can be used in adjunct to nicotine replacement therapy.

Vaccinations: all patients with COPD should be offered an annual influenza vaccination. In addition, pneumococcal vaccination should be offered according to the dosing schedules set by the director public health.

Pulmonary rehabilitation: pulmonary rehabilitation is a multidisciplinary programme of care for patients with chronic respiratory impairment that is individually tailored and designed to optimize each patient's physical and social performance and autonomy. National guidelines advocate that all patients with COPD who have an MRC score of $\geq 3$ should be referred for pulmonary rehabilitation, as should patients with an MRC score of 2 and significant breathlessness. Benefits include a reduction in exacerbation frequency and improved quality of life.

Nutrition: many patients with COPD have cachexia and a low body mass index (BMI); which has been shown to be poor prognostic marker. All COPD patients with a BMI $<20 \mathrm{~kg} / \mathrm{m}^{2}$ should be given nutritional supplements and referred for dietetic advice as per national guidance (NICE 2010).

Psychological and social support: depression is a common co-morbidity in patients with a chronic medical problem and can, along with anxiety, be considered in patients with COPD. Validated screening tools and questionnaires can be used in primary and secondary care to help provide a diagnosis of depression and anxiety. Psychological support should be provided by a multidisciplinary team; community services, including community respiratory nurses and physiotherapists, are particularly important.

Palliative care: often, despite pharmacological and non-pharmacological treatment aimed at improving lung function and reducing exacerbations, patients with severe and very severe COPD remain symptomatic; specialist palliative care services should then be considered and involved where possible. Low-dose opiates and short-acting benzodiazepines are often effective at relieving breathlessness; these, as well as social support, can be provided via the palliative care team. Plans for end-of-life care can also be discussed with patients and their families, supported in the community by specialist teams. 


\section{Key References}

1. Bafadhel, M., et al., Acute exacerbations of chronic obstructive pulmonary disease:

identification of biologic clusters and their biomarkers. Am J Respir Crit Care Med, 2011

184(6): p. 662-71.

2. Albert, R.K., et al., Azithromycin for Prevention of Exacerbations of COPD. New England Journal of Medicine, 2011. 365(8): p. 689-698.

3. Criner, G.J. and A.L. Sternberg, National Emphysema Treatment Trial: The Major Outcomes of Lung Volume Reduction Surgery in Severe Emphysema. Proceedings of the American Thoracic Society, 2008. 5(4): p. 393-405.

4. Shah, P.L. and F.J.F. Herth, Current status of bronchoscopic lung volume reduction with endobronchial valves. Thorax, 2014. 69(3): p. 280-286.

5. Scanlon, P.D., et al., Smoking cessation and lung function in mild-to-moderate chronic obstructive pulmonary disease. The Lung Health Study. Am J Respir Crit Care Med, 2000. 161(2 Pt 1): p. 381-90. 
Table 1

\begin{tabular}{|l|l|}
\hline \multicolumn{2}{|l|}{ MRC Dyspnoea Scale } \\
\hline Grade & Degree of breathlessness related to activity \\
\hline 1 & Not troubled by breathlessness except on strenuous exercise \\
\hline 2 & Short of breath when hurrying or walking up a slight hill \\
\hline 3 & $\begin{array}{l}\text { Walks slower than contemporaries on level ground because of breathlessness, or } \\
\text { has to stop for breath when walking at own pace }\end{array}$ \\
\hline 4 & Stops for breath after walking about 100 m or after a few minutes on level ground \\
\hline 5 & Too breathless to leave the house, or breathless when dressing or undressing \\
\hline
\end{tabular}

Table 2

National Institute for Health and Care Excellence COPD disease classification using spirometry

\begin{tabular}{|l|l|}
\hline Severity & FEV $_{1} \%$ predicted \\
\hline Mild (stage 1) & $\geq 80 \%$ \\
\hline Moderate (stage 2) & $<80 \% \mathrm{FEV}_{1} \geq 50 \%$ \\
\hline Severe (stage 3) & $<50 \% \mathrm{FEV}_{1} \geq 30 \%$ \\
\hline Very severe (stage 4) & $<30 \% \mathrm{FEV}_{1}$ \\
\hline
\end{tabular}


$05 / 04 / 2017$

\section{Figure 1 COPD Assessment Test ${ }^{\mathrm{TM}}$}

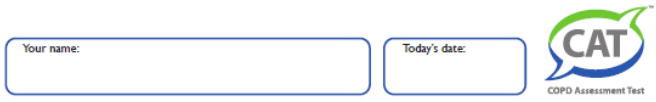

How is your COPD? Take the COPD Assessment Test ${ }^{\text {Tm }}$ (CAT)

This questionnaire will help you and your healthcare professional measure the impact COPD (Chronic Obstructine Pulmonary Disease) is having on your welleeing and dalily lite. Your answers, and test score, can be used by you and For each item below. place a mark $(X)$ in the box that best describes you currenty Be sure to only select one response For each item betow

Examplet I am very happy (2) X (2) (3) (4) (5) lam very sad

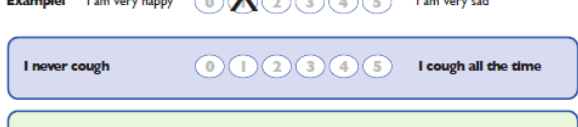

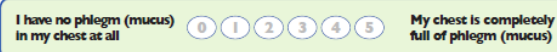

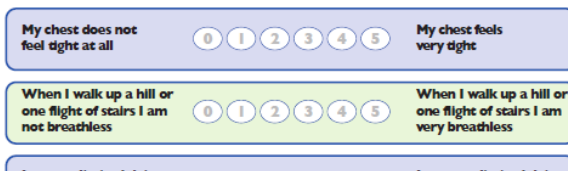

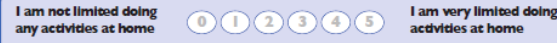

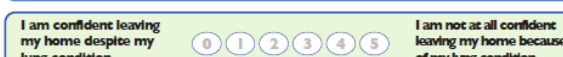

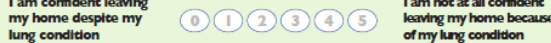

$\begin{array}{lll}\text { I sleep soundly } & \text { (1) (1) (2) (3) (4) (5) (5) } \begin{array}{l}\text { I donts sleep soundly } \\ \text { because of my lung } \\ \text { conditton }\end{array}\end{array}$

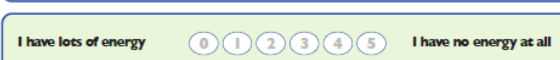

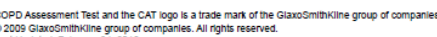

TOTAL

\section{Commented [RE1]: Authors:}

Please can you send the permission for the figure.

If any costs have been incurred, please do let us know and we will reimburse you 
05/04/2017

Figure 2

Typical chest X-ray from a patient with COPD, showing gross hyperinflation, flattened hemi-diaphragms, horizontal rib configuration and a 'stretched' cardiac silhouette.

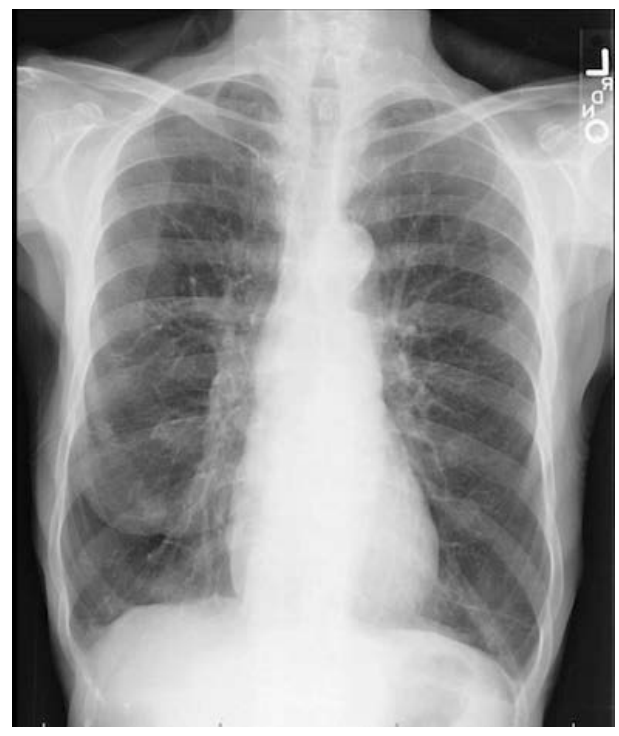


$05 / 04 / 2017$

\section{Figure 3}

Computed tomography scan of the chest showing bullous disease (black circle) in COPD.

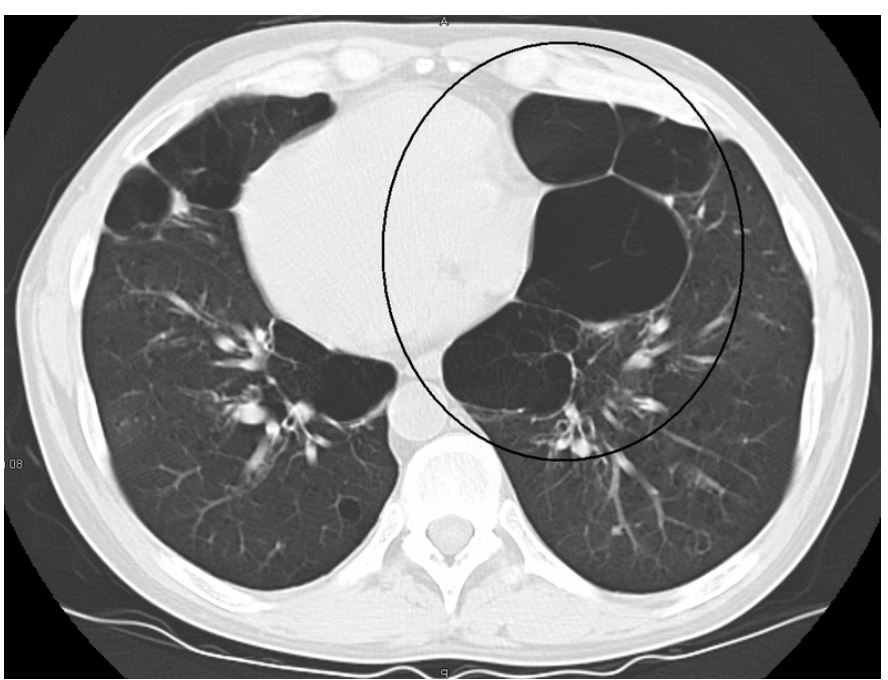

\section{Commented [FC2]: Authors:}

Is it possible to use an alternative image showing classical emphysema where the cysts are 'wall-deficient'? These cysts

have very well circumscribed walls. If this image is used in

the final manuscript, please check that the L-R orientation is correct. 
$05 / 04 / 2017$

\section{Figure 4}

Classification of COPD using GOLD 2015 guidance. mMRC, xxxxx.
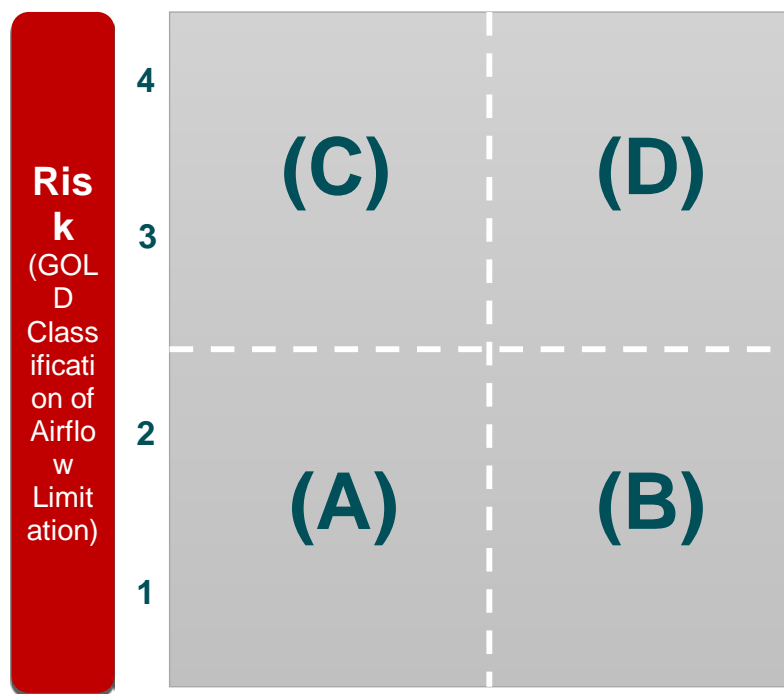

$\geq 2$

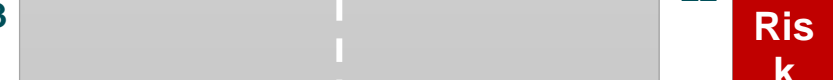

k

(Exac

erbati

on

2

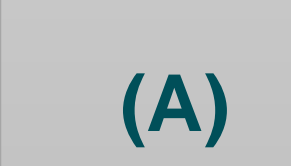

1

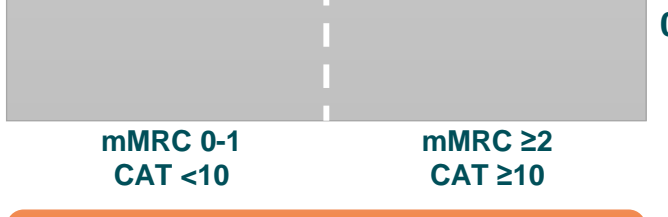

Symptoms

(mMRC or CAT score)

\section{Commented [CMW3]: Authors:}

please add definition as the ' $\mathrm{m}$ ' isn't explained in the text. Is it 'modified'?

Commented [RE4]: Authors:

Please can you send the permission for the figure.

If any costs have been incurred, please do let us know and we will reimburse you 
$05 / 04 / 2017$

\section{Figure 5}

NICE guidelines for inhaled therapy for COPD.

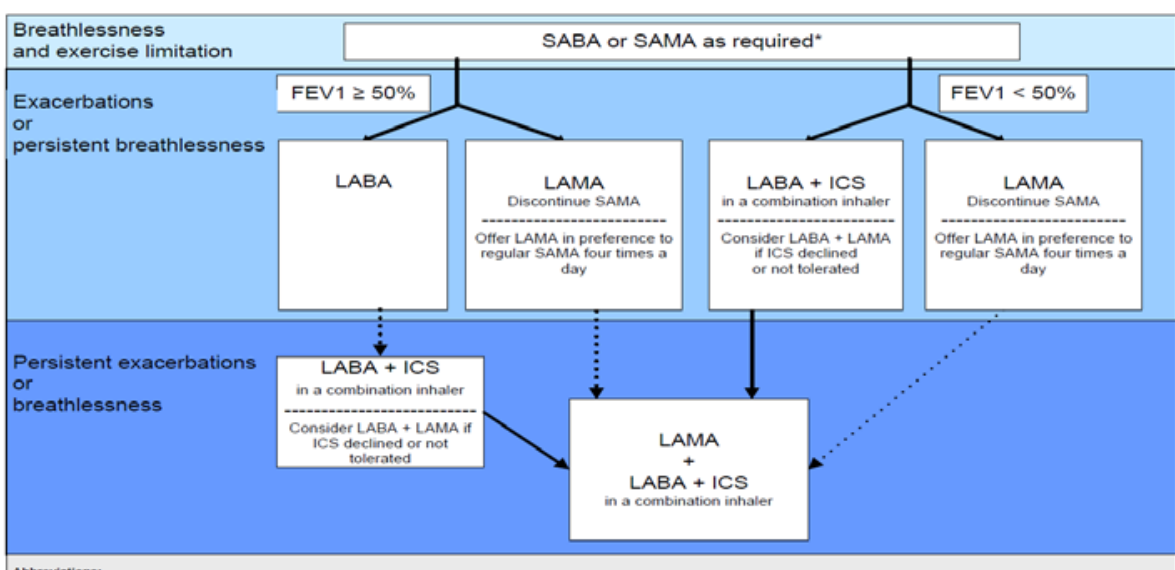

Abbreviations:

\section{Commented [RE5]: Authors:}

Is this your own figure?

If not, please can you send the permission for the figure.

If any costs have been incurred, please do let us know and we will reimburse you 